\title{
Ventricular access device infection rate: a retrospective study and review of the literature
}

\author{
Jason K. Chu • Samir Sarda • Kristina Falkenstrom • \\ William Boydston • Joshua J. Chern
}

Received: 18 June 2014 / Accepted: 1 August 2014 / Published online: 22 August 2014

(C) Springer-Verlag Berlin Heidelberg 2014

\begin{abstract}
Purpose Ventricular access devices (VAD) are often used for treatment of posthemorrhagic hydrocephalus $(\mathrm{PHH})$ in preterm infants. The reported rates of infection have varied and range from 0 to $22 \%$. The objective of our study is to present our VAD associated infection at our institution.

Methods The charts for patients that had VADs inserted between May 1, 2009 and October 31, 2013 at a single institution (Children's Healthcare of Atlanta) were retrospectively reviewed. The number of VAD infections, defined as either cerebrospinal fluid (CSF)-positive cultures or wound complication, was recorded. Of patients that survived, the number of VAD to shunt conversions was also examined. The data from 15 previously published studies were pooled to determine overall VAD infection and VAD to shunt conversion rates. Results A total of 142 VADs were placed. There were 13 infections $(9.2 \%), 11$ of which had CSF-positive cultures (7.7\%). There were two wound complications with negative CSF cultures. Six patients died after VAD placement for reasons unrelated to their VAD surgeries $(4.2 \%)$. In the remaining patients, there were $113 \mathrm{VAD}$ to shunt conversions $(83.1 \%)$. Fifteen studies that reported VAD infections were analyzed; an overall infection rate of $7.0 \%$ and VAD to shunt conversion rate of $79 \%$ were calculated.

Conclusions While VAD is a valuable tool to treat PHH, it remains a procedure with an infection rate between 7.0 and $8.0 \%$. Close follow-up is needed to capture these adverse
\end{abstract}

J. K. Chu

Department of Neurosurgery, Emory University School of Medicine, Atlanta, GA, USA

S. Sarda $\cdot$ K. Falkenstrom $\cdot$ W. Boydston $\cdot$ J. J. Chern $(\bowtie)$ Pediatric Neurosurgery Associates, Children's Healthcare of Atlanta, 5455 Meridian Mark Road, Suite 540, Atlanta, GA 30342, USA e-mail: Joshua.Chern@choa.org events as early as possible. Approximately $80 \%$ of patients with $\mathrm{PHH}$ will require permanent CSF diversion.

Keywords Posthemorrhagic hydrocephalus $\cdot$ Intraventricular hemorrhage · Ventricular access device (VAD) · Infection · Preterm infant

\section{Introduction}

Papile grade III and IV germinal matrix hemorrhage (GMH) with associated intraventricular hemorrhage (IVH) affects 15$20 \%$ of premature neonates with a birth weight of less than $1,500 \mathrm{~g}$ [1]. The pathophysiology behind GMH/IVH is thought to be fragile vasculature susceptible to rupture with fluctuations in cerebral perfusion [2]. Infants with grade III or IV GMH/IVH are prone to developing posthemorrhagic hydrocephalus $(\mathrm{PHH})$ and are at risk for further neurological injury from increased intracranial pressure (ICP).

The classic treatment for $\mathrm{PHH}$ is a ventriculoperitoneal (VP) or ventriculoatrial (VA) shunt. However, this is not always an immediate option because these patients often have multiple sequelae of neonatal prematurity that precludes shunt placement, including low birth weight, hostile abdomen, neonatal infection, and hemodynamic instability. Acetazolamide has not been proven to be effective in reducing the rate of $\mathrm{PHH}$. Serial lumbar punctures, external ventricular drainage, or ventricular punctures also carry inherent infection risks and the risk of porencephaly [1]. Alternative surgical options include the placement of temporizing devices such as ventriculosubgaleal shunt (SGS) or a subcutaneous ventricular access device (VAD). Both have been shown to be an effective temporizing measure for $\mathrm{PHH}$ and an intermediate step toward shunt placement [1]. Currently, there is little evidence to suggest one procedure is more advantageous over the other in terms of infection, wound complications, or shunt conversion 
[3]. Thus, the decision to place either an SGS or VAD is largely surgeon- and institution-dependent.

A previous report from our institution demonstrated that the VAD was a valuable tool but with significant complications, including infection, wound dehiscence, cerebrospinal fluid (CSF) leak, and device migration [4]. The overall VAD infection rate has ranged from 0 to $22 \%$ in the current literature. The purpose of our study was to examine our experience with VAD infections and to contribute to the existing literature.

\section{Methods}

A total of 150 VADs were scheduled for insertion in patients between two campuses of a single institution (Children's Healthcare of Atlanta, Scottish Rite, and Egelston campuses) between May 1, 2009 and October 31, 2013 by board-certified and board-eligible pediatric neurosurgeons and fellows. After careful review of the patient charts, eight cases were excluded because of patient death prior to VAD insertion $(n=5)$, VAD placed for diagnosis other than PHH $(n=1)$, and patients with VADs and preexisting CNS infections $(n=2$, one patient had an undocumented meningitis that was diagnosed from intraoperative CSF cultures, and a second patient was transferred to our institution with a preexisting Staphylococcus epidermidis VAD infection). Therefore, a total of 142 VADs were included in this study. At the Scottish Rite campus, all of the VADs inserted were Medtronic model no. 44101 (barium impregnated catheter, 12-mm tapping reservoir, 3.0-cm-length intracranial catheter, $1.2 \mathrm{~mm}$ inner diameter, $2.1 \mathrm{~mm}$ outer diameter). Medtronic model no. 21029 (barium impregnated catheter, $12 \mathrm{~mm}$ tapping reservoir, 23-cm-length intracranial catheter that is trimmed to appropriate length, $1.2 \mathrm{~mm}$ inner diameter, $2.1 \mathrm{~mm}$ outer diameter) was utilized for all the VADs at the Egelston campus.

Patient demographics were collected from the hospital electronic medical record. Gestational age, birth weight, VAD insertion date, weight at VAD insertion, intubation status, VAD insertion location (neonatal intensive care unit (NICU) versus OR), VAD infection date and VAD to shunt conversion date, and future shunt infection were all documented. A VAD infection was defined as either CSF-positive cultures, wound infection, or wound dehiscence with exposed hardware that necessitated removal of the VAD. Once a VAD infection was documented, it was removed within $24 \mathrm{~h}$. In addition to the infection rate, two clinical endpoints were recorded after a VAD was placed: conversion to a shunt (either VP or VA) and in hospital mortality.

A literature review was conducted to identify previous studies with documented VAD infections. A search was conducted on PubMed/MEDLINE for the terms: "preterm infant," "ventricular access device" or "VAD," "intraventricular reservoir" or "ventricular reservoir," "intraventricular hemorrhage," "infection," "post-hemorrhagic hydrocephalus" or "posthemorrhagic hydrocephalus" in either the title, abstract, or keywords. Relevant articles were reviewed for VAD infection as well as shunt conversion rate.

\section{Results}

There were 142 VADs inserted during the 4-year study period, and population demographics are summarized in Table 1. All of the patients were preterm and very low or extremely lowbirth weight infants. The average birth age was $28 \pm 3.2$ weeks, and the average birth weight was $1,033.8 \pm 489.3 \mathrm{~g}$. The average weight at VAD insertion was $1,361.0 \pm 485.8 \mathrm{~g}$, and this was significantly increased compared to the patient's average birth weight (paired $t$ test, $p<0.001$ ). Subgroup analysis did not reveal any differences in weight between the VAD infection and noninfected groups, regardless if it was birth weight

Table 1 Patient demographics

\begin{tabular}{ll}
\hline Total number of VADs placed & 142 \\
Average gestational age $( \pm \mathrm{STD})$ & $27.0 \pm 3.2$ weeks \\
Average birth weight $( \pm \mathrm{STD})$ & $1,033.8 \pm 489.3 \mathrm{~g}^{*}$ \\
Average weight at VAD placement $( \pm \mathrm{STD})$ & $1,361.0 \pm 485.8 \mathrm{~g}^{*}$ \\
VAD infections & $13(9.2 \%)$ \\
CSF-positive cultures & $11(7.7 \%)$ \\
$\quad$ Staphylococcus epidermidis & 4 \\
Enterococcus faecalis & 2 \\
$\quad$ Escherichia coli & 2 \\
Pseudomonas aeruginosa & 2 \\
Enterobacter cloacae & 1 \\
Wound complications (negative CSF cultures) & $2(1.4 \%)$ \\
Median time from VAD insertion to infection & 19 days \\
& (range, $7-63$ days) \\
Shunts placed after VAD infection & 11 \\
$\quad$ VP shunt & 10 \\
$\quad$ VA shunt & 1 \\
Died (no shunt placed) & 2 \\
VAD placed but died before shunt placement & $6(4.2 \%)$ \\
(including infection patients, $n=2)$ & \\
Median time from VAD insertion to death & 50 days \\
Surviving VAD patients converted to shunts & $113(83.1 \%)$ \\
(including infection patients, $n=11)$ & 109 \\
VP shunt & 4 \\
VA shunt & 56 days \\
Median time for VAD to shunt conversion & (range, $3-549$ days) \\
Surviving VAD patients that did not & $23(16.9 \%)$ \\
require shunts & \\
\hline
\end{tabular}

${ }^{*} p<0.001$ (paired $t$ test) 
or weight at VAD insertion (unpaired $t$ test, $p=0.91$ birth weights; $p=0.88$ weight at VAD insertion)

There were a total of 13 VAD infections, and their demographics are shown in Table 2. This corresponds to an overall incidence of $9.2 \%$. Eleven of these had documented CSFpositive cultures $(7.7 \%)$ and two were wound complications (1.4\%, 1 infection, 1 dehiscence). The median time from VAD insertion to VAD removal was 19 days and ranged from 7 to 63 days post-VAD insertion. The pathogens responsible for VAD infections were $S$. epidermidis $(n=4)$, Enterococcus faecalis $(n=2)$, Escherichia coli $(n=2)$, Pseudomonas aeruginosa $(n=2)$, and Enterobacter cloacae $(n=1)$. Both patients with wound complications had negative CSF cultures. Treatment of all VAD infections necessitated removal of the VAD, with or without external ventricular drain (EVD) placement, as well as antibiotics as dictated by the Infectious Disease Team (see Table 2 for details). Reimplantation of CSF diversion devices (VAD replacement or a shunt) was done after serial CSF cultures were sterile and after clearance from the Infectious Disease Team. Three patients developed secondary CNS infections related to their EVD, which was treated with EVD replacement and antibiotics. Two patients in this group died before a shunt was placed for causes unrelated to their VAD infections: one patient had care withdrawn in the NICU, while the second was transferred to hospice. The remaining 11 patients required a shunt (VP shunt, $n=10$; VA shunt, $n=1$ ).

Of the 142 patients with VAD insertion, there were 6 (4.2\%) patients who had a VAD placed and died from other sequelae of prematurity before conversion to shunt (including the two VAD infection patients). In these patients, the median length of survival after VAD insertion was 50 days, ranging from 8 to 168 days. In the remaining patients, the VAD to shunt conversion rate was $83.1 \%(113 / 136$ patients, including the 11 patients with VAD infection). The majority of these were VP shunts $(n=109)$ and only a small proportion were VA shunts $(n=4)$. The median time from VAD placement to conversion to a shunt was 56 days, ranging from 3 to 549 days. The remaining 23 patients ( $16.9 \%$ ) did not require conversion to a shunt at a minimal follow-up of 6 months.

Table 3 depicts secondary data analysis for risk factors that may be related to the development of VAD infections. When examining intubation status, a total of $55(38.7 \%)$ patients were intubated at the time of VAD insertion. In the VAD infection group, seven $(53.8 \%)$ patients were intubated at the time of VAD insertion and six $(46.2 \%)$ were not. In the noninfected group, 48 (37.2\%) were intubated and 81 were not $(62.8 \%)$. Intubation status was not identified as a significant risk factor contributing to VAD infection $\left(\chi^{2}=1.377\right.$; $d f=1$; Fisher's exact test, $p=0.250$ ).

The majority of our VAD insertions were placed in the operating room (125/142 patients, $88.0 \%)$. There were a total of 17 VADs placed in the NICU, and this was institutionally dependent, as all were done at the CHOA Egelston campus. Of the 17 VADs that were placed in the NICU, three became infected, which corresponds to $17.6 \%$ of VADs placed in NICU and $23.1 \%$ of the total VAD infections. VAD insertion location was also not identified as a statistically significant risk factor contributing to $\mathrm{VAD}$ infection $\left(\chi^{2}=2.072 ; d f=1\right.$; Fisher's exact test, $p=0.161$ ).

Our overall shunt infection rate after VAD placement was $6.2 \%$ (7/113 patients). Subgroup analysis revealed that there were two patients with VAD infections prior to shunt placement $(18.1 \%$ of the VAD infection population that were shunted). The remaining five patients did not have a VAD infection but developed a future shunt infection $(4.9 \%$ of the noninfected population). VAD infection before shunt placement was not a significant risk factor for developing shunt infection $\left(\chi^{2}=3.013 ; d f=1\right.$; Fisher's exact test, $\left.p=0.138\right)$.

\section{Discussion}

\section{VAD infection rate}

The use of VADs to treat PHH in preterm infants was first introduced by McComb in the early 1980s, and numerous studies have suggested that it is an effective bridge to permanent CSF diversion in this population. However, the reported VAD infection rate has considerable variation in the literature, ranging from 0 to $22 \%$. The small sampling size of these studies ( $n=12$ to 149 ) directly contribute to the wide range of complication rates. Table 4 depicts the VAD infection rate reported in the current literature. A total of 15 studies from 1983 to 2012 were found through PubMed and included. The numbers of patients and VAD infections were then combined for a total of 725 patients and 51 reported infections. The infection rate from these pooled data was approximately $7.0 \%$.

Our study suggests an overall VAD infection rate of $9.2 \%$, and in those with proven CSF-positive cultures, the infection rate was $7.7 \%$. This is comparable to a previous report from our institution by Hudgins et al., who has the largest documented study population with 149 patients and a CSF-positive infection rate of $8.1 \%$ [5]. Overall, our institutional experience is similar to what is reported in the literature and suggests that the incidence of VAD infections is between 7 and $8 \%$. Interestingly, our study did not find that differences in weight (birth or at VAD insertion), intubation status, or VAD placement location as statistically significant risk factors associated with the development of VAD infections. Identifying potential risk factors for VAD infection should be an aim for future studies.

One risk factor not examined in our study was the effect of antibiotic-coated ventricular catheters on VAD infection rates. The current literature suggests that antibiotic-coated 


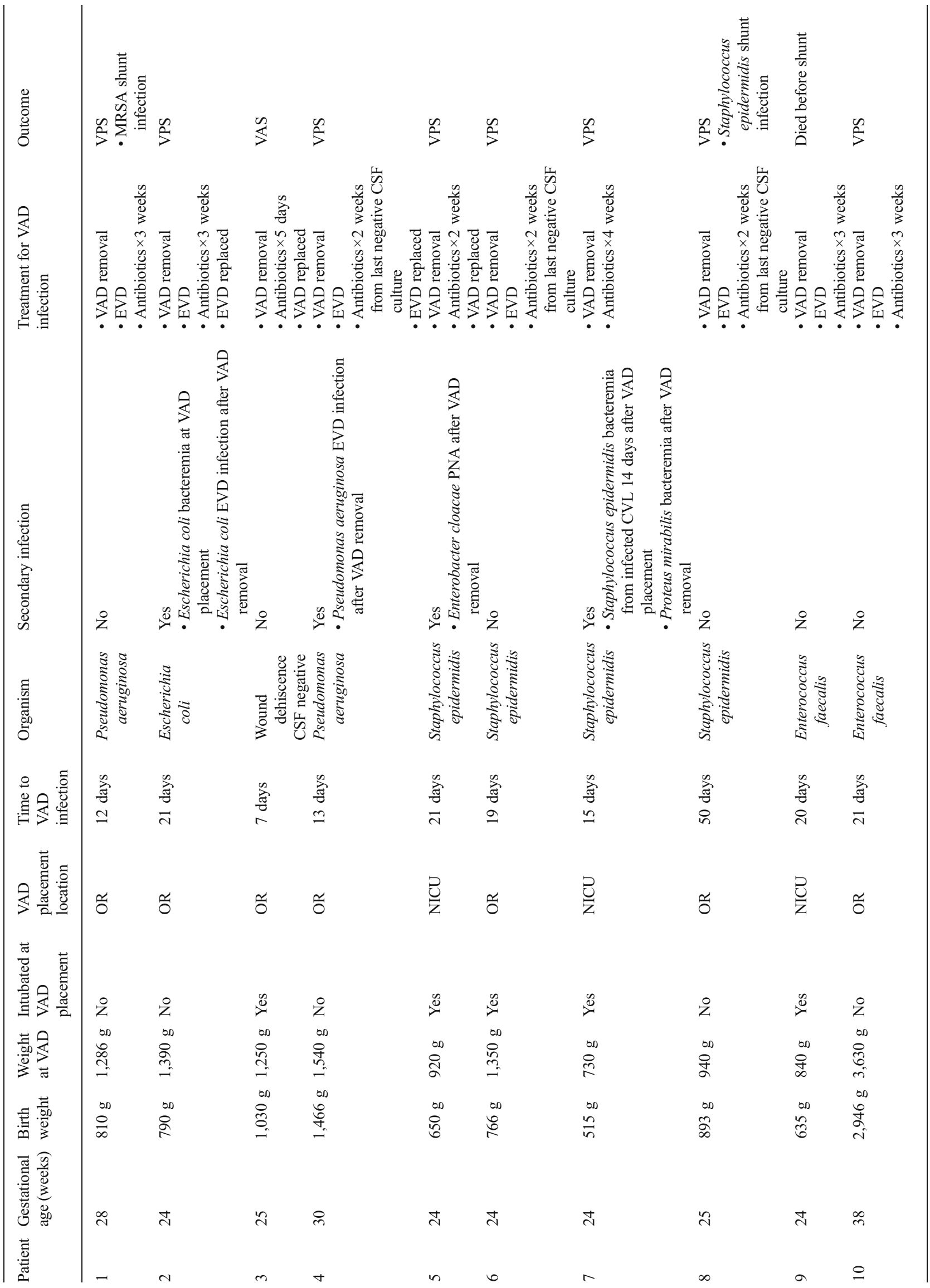


ventricular catheters for EVDs and shunts reduce the incidence of ventriculitis $[6,7]$. It stands to reason that there would be a similar effect for VADs. At our institution, VADs are supplied by Medtronic and their models are accompanied with barium impregnated catheters. Our CHOA campuses utilize different VAD models (no. 44101 at Scottish Rite and no. 21029 at Egelston); one notable difference between these two models is that no. 44101 has the ventricular catheter built into the reservoir, whereas no. 21029 requires the surgeon to attach a ventricular catheter to the reservoir. Therefore, it would be possible to utilize an antibiotic-impregnated ventricular catheter on model no. 21029 and will require future investigations to address this question.

Over the last 30 years, the incidence of VAD infections has decreased and partly attributed to improved ICU care in between taps [8]. A second reason is the strict adherence of sterile technique when device access is required. In a study of 29 patients, Kormanik et al. demonstrated that the use of sterile technique for repeated VAD access did not increase risk of ventriculitis [9]. Their VADs were prepped with iodine and accessed a total of 681 times with sterile equipment (gloves, mask, butterfly needles); there were no reported infections, despite $45 \%$ of their patients having blood culture proven sepsis. While the utility of surveillance CSF samples remains controversial, CSF WBC count, neutrophil proportion, and protein count are sensitive markers that can aid in the diagnosis of early infection [10].

Patients with high grade IVH that develop PHH often have permanent neurological deficits and have been shown to have poorer functional outcomes despite treatment $[1,11]$. As perinatal meningitis has been shown to also negatively affect neurodevelopmental outcome $[12,13]$, the avoidance of a secondary, infectious CNS insult is favorable. Therefore, care providers must be vigilant in preventing VAD-associated nosocomial CNS infections as it not only represents a source of avoidable patient morbidity but also has long-term neurological consequences.

\section{VAD to shunt conversion rate}

For our infants with PHH and VADs that survived, $83.1 \%$ of our patients required conversion of their VAD to a shunt. Table 4 also depicts the pooled data for VAD to shunt conversion rates from 15 studies: the combined number of surviving patients with a VAD was 578 and the total number of patient's that required conversion of a VAD to a shunt was 454 . This corresponds to a conversion rate of $79 \%$. This is in concordance with previously reported data from larger population studies and range from 73 to $88 \%[3,5]$. Our overall shunt infection rate after VAD conversion was $6.2 \%$. This in agreement with previous cohorts that report an infection rate of 6 $8 \%$ but lower than a recently published, large multicenter study that reported an infection rate of $11.7 \%$ per patient [14]. 
Table 3 Secondary analysis of risk factors that may be associated with VAD infections

\begin{tabular}{llc}
\hline Risk factor & VAD infection & No VAD infection \\
\hline Average birth weight $( \pm \mathrm{STD})$ & $1,019.1 \pm 640.9 \mathrm{~g}$ & $1,035.3 \pm 474.6 \mathrm{~g}$ \\
& Unpaired $t$ test, $p=0.91$ & \\
Average weight at VAD placement $( \pm \mathrm{STD})$ & $1,380.5 \pm 726.3 \mathrm{~g}$ & $1,359.0 \pm 458.8 \mathrm{~g}$ \\
& Unpaired $t$ test, $p=0.88$ & \\
Intubated at VAD placement & 7 & 48 \\
Not intubated at VAD placement & 6 & 81 \\
& $\chi^{2}=1.377 ; d f=1$; Fisher's exact test, $p=0.250$ \\
VAD placed in NICU & 3 & 14 \\
VAD placed in OR & 10 & 115 \\
& $\chi^{2}=2.072 ; d f=1$; Fisher's exact test, $p=0.161$ \\
Shunt infection & 2 & 5 \\
No shunt infection & 9 & 97 \\
& $\chi^{2}=3.013 ; d f=1 ;$ Fisher's exact test, $p=0.138$ \\
\hline
\end{tabular}

The risk of developing a shunt infection is strongly correlated with increasing number of revision surgeries [15]. In our study, a treated VAD infection prior to shunt placement was not a risk factor for developing shunt infection in the future.

In the absence of a hostile abdomen, we favor placement of a VP shunt over a VA shunt given the early need for revision (secondary to distal catheter length limitations) as well as the high morbidity associated with VA shunt infections. Multiple studies have demonstrated that early shunting in preterm $\mathrm{PHH}$ patients are fraught with complications, including high postoperative infection rates and early revision rates [5]. Lower birth weight as well as gestational age are identifiable risk factors for early shunt revisions $[16,17]$ and must be taken into consideration when deciding between a temporizing procedure (VAD or VSG) versus permanent CSF diversion.

Recently, Romero et al. advocated the use of VP shunt placement as a first line and definitive therapy for infants with symptomatic PHH [18]. They used a stringent definition for symptomatic hydrocephalus as well as a strict patient selection for VP shunt placement, including a weight of greater than

Table 4 Published VAD infection and conversion rates

\begin{tabular}{|c|c|c|c|c|c|c|c|}
\hline Author & Year & No. of patients & Infections & Infection rate $(\%)$ & No. of survivors & No. of shunts & Conversion rate $(\%)$ \\
\hline McComb [19] & 1983 & 20 & 0 & 0.0 & 7 & 7 & 100 \\
\hline Anwar [20] & 1986 & 19 & 2 & 10.5 & 18 & 15 & 83 \\
\hline Gaskill [21] & 1988 & 38 & 0 & 0.0 & 30 & 30 & 100 \\
\hline Marlin [22] & 1988 & 12 & 2 & 16.7 & 38 & 33 & 87 \\
\hline Brockmeyer [23] & 1989 & 20 & 2 & 10.0 & 16 & 12 & 75 \\
\hline Leonhardt [24] & 1989 & 13 & 0 & 0.0 & 13 & 12 & 92 \\
\hline Levy [25] & 1997 & 72 & 2 & 2.8 & 53 & 47 & 89 \\
\hline Hudgins [5] & 1998 & 149 & 12 & 8.1 & 133 & 117 & 88 \\
\hline Richard [11] & 2001 & 64 & 14 & 21.9 & 45 & 31 & 69 \\
\hline \multirow[t]{2}{*}{ Brouwer [8] } & 2007 & 26 & 5 & 19.2 & 26 & 12 & 46 \\
\hline & & 50 & 2 & 4.0 & 50 & 22 & 44 \\
\hline Lam [26] & 2009 & 16 & 0 & 0.0 & 16 & 15 & 94 \\
\hline Willis [17] & 2009 & 15 & 1 & 6.7 & 13 & 12 & 92 \\
\hline Limbrick [3] & 2010 & 65 & 4 & 6.2 & 64 & 49 & 77 \\
\hline Kormanik [9] & 2010 & 29 & 0 & 0.0 & 29 & 21 & 72 \\
\hline Bajaj [10] & 2012 & 52 & 5 & 9.6 & NR & NR & NR \\
\hline Tian [27] & 2012 & 27 & 0 & 0.0 & 27 & 19 & 70 \\
\hline Total & & 725 & 51 & 7.0 & 578 & 454 & 79 \\
\hline Current study & 2014 & 142 & 13 & 9.2 & 136 & 113 & 83 \\
\hline
\end{tabular}

$N R$ not reported 
$1,500 \mathrm{~g}$ prior to shunt insertion. Out of 139 patients with grade III/IV IVH, only 47 shunts were inserted. The authors suggested that the remaining 92 patients $(66 \%)$ did not require surgical treatment for their "ventriculomegaly." However, whether or not this subset of patients required a form CSF diversion as well as their outcomes are not reported. Nonetheless, they report good long-term functional outcomes in their shunted patients and suggest that avoidance of temporizing procedures decreases overall patient morbidity. The placement of a shunt as primary treatment for $\mathrm{PHH}$ without previous temporizing procedures is an interesting concept and its efficacy is yet to be fully elucidated.

Our study also suggests that VAD placement with intermittent CSF drainage was sufficient to halt hydrocephalus in $16.9 \%$ of patients. Previous reports suggest that interment VAD tapping can prevent the development of PHH in 12$31 \%$ of patients and is thought to be related to restoration of CSF absorption by clearing obstructive hemorrhagic material from the CSF [3, 5, 11, 17]. Our pooled data suggests that the use of a VAD can prevent the need for a shunt in approximately $21 \%$ of patients. Whether increased frequency of tapping or larger tapping volumes prevents the development of PHH will require future investigation.

\section{Conclusions}

VADs are a reasonable alternative for treatment of PHH in patients who are unable to have a shunt placed. The infection rate after VAD placement is approximately 7 to $8 \%$. Although this is lower than some previously reported data, it remains a procedure with a significant infection rate. Close follow-up and the use of aseptic technique when the VAD is accessed are critical in minimizing iatrogenic infection. While a VAD has the potential of halting the need for permanent CSF diversion in PHH, the vast majority of patients will require a shunt when medically stable. These are important statistics when counseling families about the risks and benefits of VAD placement.

Conflict of interest The authors declare that there are no conflicts of interest.

\section{References}

1. Robinson S (2012) Neonatal posthemorrhagic hydrocephalus from prematurity: pathophysiology and current treatment concepts. J Neurosurg Pediatr 9:242-258

2. Shooman D, Portess H, Sparrow O (2009) A review of the current treatment methods for posthaemorrhagic hydrocephalus of infants. Cerebrospinal Fluid Res 6:1
3. Limbrick DD Jr, Mathur A, Johnston JM, Munro R, Sagar J, Inder T, Park TS, Leonard JL, Smyth MD (2010) Neurosurgical treatment of progressive posthemorrhagic ventricular dilation in preterm infants: a 10-year single-institution study. J Neurosurg Pediatr 6:224-230

4. Bot G, Constantini S, Roth J (2013) Intraventricular migration of ventricular access device. Child's Nerv Syst : ChNS : off J Int Soc Pediatr Neurosurg 29:1975-1976

5. Hudgins RJ, Boydston WR, Gilreath CL (1998) Treatment of posthemorrhagic hydrocephalus in the preterm infant with a ventricular access device. Pediatr Neurosurg 29:309-313

6. Wright K, Young P, Brickman C, Sam T, Badjatia N, Pereira M, Connolly ES, Yin MT (2013) Rates and determinants of ventriculostomy-related infections during a hospital transition to use of antibiotic-coated external ventricular drains. Neurosurg Focus 34: E12

7. Thomas R, Lee S, Patole S, Rao S (2012) Antibiotic-impregnated catheters for the prevention of CSF shunt infections: a systematic review and meta-analysis. Br J Neurosurg 26:175-184

8. Brouwer AJ, Groenendaal F, van den Hoogen A, Verboon-Maciolek M, Hanlo P, Rademaker KJ, de Vries LS (2007) Incidence of infections of ventricular reservoirs in the treatment of post-haemorrhagic ventricular dilatation: a retrospective study (1992-2003). Arch Dis Child Fetal Neonatal Ed 92:F41-43

9. Kormanik K, Praca J, Garton HJ, Sarkar S (2010) Repeated tapping of ventricular reservoir in preterm infants with post-hemorrhagic ventricular dilatation does not increase the risk of reservoir infection. J Perinatol : off J Calif Perinatal Assoc 30:218-221

10. Bajaj M, Lulic-Botica M, Natarajan G (2012) Evaluation of cerebrospinal fluid parameters in preterm infants with intraventricular reservoirs. J Perinatol : off J Calif Perinatal Assoc 32:786-790

11. Richard E, Cinalli G, Assis D, Pierre-Kahn A, Lacaze-Masmonteil T (2001) Treatment of post-haemorrhage ventricular dilatation with an Ommaya's reservoir: management and outcome of 64 preterm infants. Child's Nerv Syst : ChNS : off J Int Pediatr Neurosurg 17:334-340

12. Adams-Chapman I, Stoll BJ (2006) Neonatal infection and long-term neurodevelopmental outcome in the preterm infant. Curr Opin Infect Dis 19:290-297

13. van Vliet EG, de Kieviet JF, Oosterlaan J, van Elburg RM (2013) Perinatal infections and neurodevelopmental outcome in very preterm and very low-birth-weight infants: a meta-analysis. JAMA Pediatr 167:662-668

14. Simon TD, Hall M, Riva-Cambrin J, Albert JE, Jeffries HE, Lafleur B, Dean JM, Kestle JR, Hydrocephalus Clinical Research N (2009) Infection rates following initial cerebrospinal fluid shunt placement across pediatric hospitals in the United States. Clinical article. J Neurosurg Pediatr 4:156-165

15. Simon TD, Butler J, Whitlock KB, Browd SR, Holubkov R, Kestle JR, Kulkarni AV, Langley M, Limbrick DD Jr, Mayer-Hamblett N, Tamber M, Wellons JC 3rd, Whitehead WE, Riva-Cambrin J, Hydrocephalus Clinical Research N (2014) Risk factors for first cerebrospinal fluid shunt infection: findings from a multi-center prospective cohort study. J Pediatr 164(1462-1468):e1462

16. Chittiboina P, Pasieka H, Sonig A, Bollam P, Notarianni C, Willis BK, Nanda A (2013) Posthemorrhagic hydrocephalus and shunts: what are the predictors of multiple revision surgeries? J Neurosurg Pediatr 11:37-42

17. Willis B, Javalkar V, Vannemreddy P, Caldito G, Matsuyama J, Guthikonda B, Bollam P, Nanda A (2009) Ventricular reservoirs and ventriculoperitoneal shunts for premature infants with posthemorrhagic hydrocephalus: an institutional experience. J Neurosurg Pediatr 3:94-100

18. Romero L, Ros B, Rius F, Gonzalez L, Medina JM, Martin A, Carrasco A, Arraez MA (2014) Ventriculoperitoneal shunt as a primary neurosurgical procedure in newborn posthemorrhagic hydrocephalus: report of a series of 47 shunted patients. Child's Nerv Syst : ChNS : off J Int Soc Pediatr Neurosurg 30:91-97 
19. McComb JG, Ramos AD, Platzker AC, Henderson DJ, Segall HD (1983) Management of hydrocephalus secondary to intraventricular hemorrhage in the preterm infant with a subcutaneous ventricular catheter reservoir. Neurosurgery 13:295-300

20. Anwar M, Doyle AJ, Kadam S, Hiatt IM, Hegyi T (1986) Management of posthemorrhagic hydrocephalus in the preterm infant. J Pediatr Surg 21:334-337

21. Gaskill SJ, Marlin AE, Rivera S (1988) The subcutaneous ventricular reservoir: an effective treatment for posthemorrhagic hydrocephalus. Child's Nerv Syst : ChNS : off J Int Soc Pediatr Neurosurg 4:291-295

22. Marlin AE, Rivera S, Gaskill SJ (1988) Treatment of posthemorrhagic ventriculomegaly in the preterm infant: use of the subcutaneous ventricular reservoir. Concepts Pediatr Neurosurg 8:15-22

23. Brockmeyer DL, Wright LC, Walker ML, Ward RM (1989) Management of posthemorrhagic hydrocephalus in the low-birthweight preterm neonate. Pediatr Neurosci 15:302-207
24. Leonhardt A, Steiner HH, Linderkamp O (1989) Management of posthaemorrhagic hydrocephalus with a subcutaneous ventricular catheter reservoir in premature infants. Arch Dis Child 64:24-28

25. Levy ML, Masri LS, McComb JG (1997) Outcome for preterm infants with germinal matrix hemorrhage and progressive hydrocephalus. Neurosurgery 41:1111-1117, discussion 1117-1118

26. Lam HP, Heilman CB (2009) Ventricular access device versus ventriculosubgaleal shunt in post hemorrhagic hydrocephalus associated with prematurity. J Matern Fetal Neonatal Med : Off J Eur Assoc Perinatal Med Fed Asia Ocean Perinatal Soc Int SocPerinatal Obstet 22:1097-1101

27. Tian AG, Hintz SR, Cohen RS, Edwards MS (2012) Ventricular access devices are safe and effective in the treatment of posthemorrhagic ventricular dilatation prior to shunt placement. Pediatr Neurosurg 48:13-20 